\title{
Late Retrieval of the Leadless Micra Transcatheter Pacemaker System
}

Kentaro Minami ${ }^{1 *}$, Jan Petrů ${ }^{1}$, Milan Chovanec ${ }^{1}$, Jan Sorf ${ }^{2}$, Pavel Hála ${ }^{1}$, Marek Janotka ${ }^{1}$, Jan Škoda ${ }^{1}$, Lucie Šedivá1 ${ }^{1}$ Vivek Y. Reddy ${ }^{3}$ and Petr Neužil ${ }^{1}$,

${ }^{1}$ Na Homolce Hospital, Prague, Czech Republic,

${ }^{2}$ Medtronic Minneapolis USA,

${ }^{3}$ Icahn School of Medicine at Mount Sinai, New York, New York, USA

*Corresponding Author: Kentaro Minami, Na Homolce Hospital, Roentgenova 37/2, 15030, Praha 5, Czech Republic.

Received Date: February 02, 2021; Accepted Date: March 11, 2021; Published Date: March 17, 2021

Citation: Kentaro Minami, Jan Petrů, Milan Chovanec, Jan Sorf, Pavel Hála, (2021) Late Retrieval of the Leadless Micra Transcatheter Pacemaker System. J. Clinical Cardiology and Cardiovascular Interventions, 4(6); Doi:10.31579/2641-0419/138

Copyright: (c) 2021 Kentaro Minami, This is an open-access article distributed under the terms of the Creative Commons Attribution License, which permits unrestricted use, distribution, and reproduction in any medium, provided the original author and source are credited.

\begin{abstract}
Aim: We report our single-center experience with the retrieval and replacement of the chronically implanted Micra transcatheter pacing system (TPS).

Material and Methods: We included 6 patients with an implanted the Micra TPSs who subsequently underwent transvenous method of retrieval at our institution. The indication for device retrieval was pacemaker syndrome in two patients, battery depletion in three patients, and need for upgrade to biventricular pacing in one patient.

Results: After an implantation duration of $555 \pm 373$ days, the overall retrieval success rate was $83.3 \%, 5$ of 6 patients. No procedure-related adverse device events occurred. In the single patient with unsuccessful retrieval, intracardiac echocardiography revealed that the Micra TPS was embedded within the cardiac wall and surrounding tissue. After retrieval, four patients received a new Micra TPS.

Conclusions: Late retrieval of an implanted Micra TPS was safe and feasible, which indicates the possibility for their safe and elective replacement with a new leadless pacing device.

Keywords: leadless cardiac pacemaker; pacemaker retrieval; micra transcatheter pacing system

Short title: micra transcatheter pacemaker retrieval
\end{abstract}

\section{Introduction}

Leadless cardiac pacemakers (LCPs) are safe, effective alternatives to conventional transvenous pacemakers for patients who require singlechamber ventricular pacing [1,2]. The Micra transcatheter pacing system (TPS) (Medtronic, Minneapolis, MN, USA), which is currently the only commercially available LCP for clinical practice, exhibited a high implantation success rate and stable pacing performance in global prospective studies [3]. Despite these promising results, the ability to retrieve a long-standing implanted TPS may be a useful management strategy in certain scenarios. With an alternative LCP of different size and shape, it has been demonstrated that retrievability is possible even after implant durations of up to 6 years, with success rates ranging between 80 $90 \%$ [4-6]. However, that LCP was removed from clinical use because of a battery dysfunction, and its longer and thinner profile (than the TPS device) precludes one from extrapolating from this experience to the feasibility, safety or efficacy of TPS retrievability. Although some successful retrieval experiences in the acute phase have been reported for the TPS device, retrieval of a long-standing implanted TPS devices has not been well-studied [7]. Therefore, we report our single-center clinical experiences regarding the retrieval of chronically implanted Micra TPS.

\section{Materials and Methods}

The present study included six patients ( 2 females) who underwent active fixation of the Micra TPS to the right ventricle (RV) in our institution between September 2014 and June 2018. The Micra TPS implant technique has been previously described. [1] Demographic data were obtained from the medical records. The study protocol was approved by the Institution Research and Ethics Committee of Na Homolce Hospital and was carried out in accordance with the Declaration of Helsinki. The basic principle regarding the possibility of retrieval or abandon of the Micra TPS was clearly explained to all the patients. Only the patients who strongly desired to be performed the retrieval of the implanted old Micra 
TPS were enrolled in this study. Written informed consent was obtained from each patient.

Retrieval success was defined as complete removal of the LCP [4]. The occurrence of any serious adverse events within the first 30 days after device retrieval was recorded. A standard definition of serious adverse event was utilized: any device- or procedure-related untoward medical occurrence leading to death or a deterioration in the patient's health, that resulted in life-threatening illness or injury, permanent impairment of a body structure or a body function, prolonged inpatient of prolonged hospitalization or medical or surgical intervention to prevent lifethreatening illness or injury or permanent impairment to a body structure or a body function [5].

The features of the TPS are shown in Figure 1.

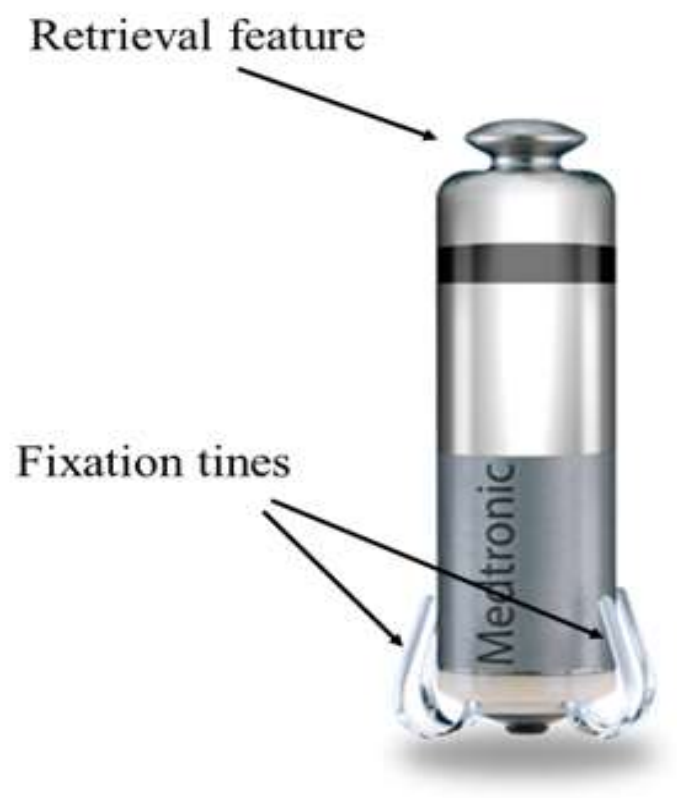

Figure 1: Micra transcatheter pacing system (TPS) *provided from Medtronic.

It has four self-expanding tines which are designed to provide a robust holding force within the cardiac tissue of right ventricular (RV). The tines are separate from the pacing electrode, which allows stable pacing performance and avoids cardiac tissue injury. The device was designed for acute retrieval capability: the proximal portion of the TPS contains a retrieve feature that can be snared, and the operators must engage the proximal retrieve feature via the snare.

All retrieval procedures were performed by two experienced operators. The retrieval catheter system was inserted via the right femoral vein accessed using the 23 Fr sheath employed for TPS insertion. Since there is no dedicated system for TPS retrieval, we used the regular delivery catheter system for TPS implantation. Prior to retrieval, a contrast agent was injected through a pigtail catheter to determine the LCP position. An intracardiac echocardiography (ICE) catheter was advanced to the right atrium using a left femoral venous approaching during all retrieval procedures to identify LCP movement, tricuspid valve damage, and the presence of pericardial effusion. For pacemaker-dependent patients, a temporary pacing lead was placed within the RV through the left femoral vein during the retrieval procedure.

A single-loop $7 \mathrm{~mm}$ snare wire (Amplatz Goose Neck Microsnare, ev3Inc., Plymouth, MN, USA) was inserted through the central lumen of the TPS delivery catheter, which in turn was subsequently introduced through the 23 Fr sheath. This "retrieval system" was advanced under fluoroscopy and ICE to the junction of the inferior vena cava and right atrium. The system's distal cone was manoeuvred into the RV to the proximal aspect of the device. The snare was advanced and deployed around the proximal retrieval feature of the TPS. After confirming coaxial alignment between the snare and the retrieval feature with multi-plane fluoroscopy, the snare was closed and locked around the retrieval feature. The snare loop was then tightened to hold the device firmly, and tension was applied, along with countertraction force from the distal cone, to release the tines from the myocardium, thereby allowing withdrawal of the device into the distal cone. The delivery catheter and Micra TPS were then withdrawn into the introducer sheath and removed from the body (Figure 2, Supplementary Online Videos A B, and C). 

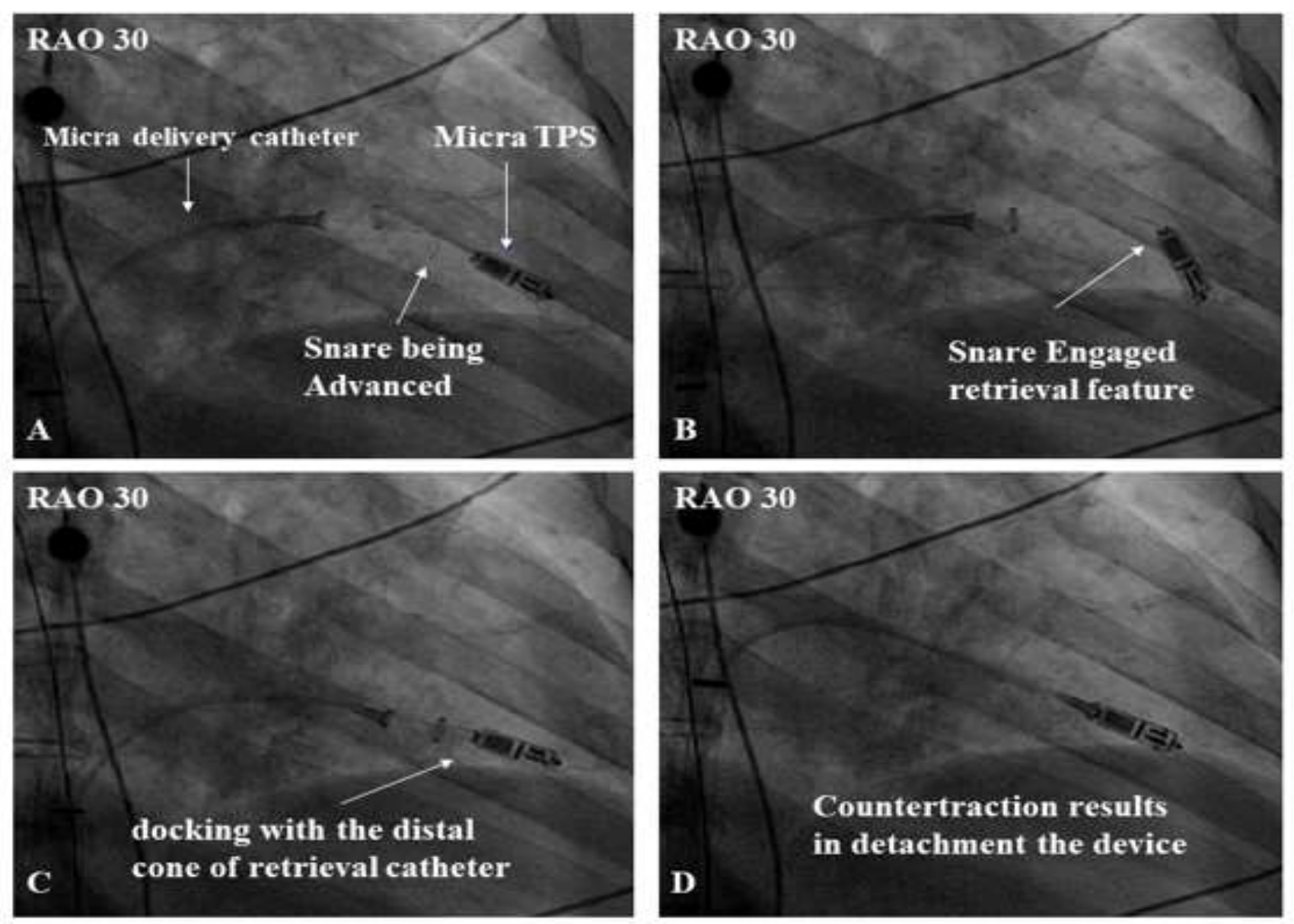

Figure 2. Fluoroscopic views of leadless pacemaker retrieval. A) With the snare advanced, the distal cone of the retrieval catheter (Micra delivery catheter) was deployed around the proximal retrieval feature of the Micra transcatheter pacing system (TPS). B) The proximal retrieval feature was snared by closing the loop, $C \& D)$ The Micra TPS was docked with the cup of the retrieval catheter, and then the constant contratraction resulted in the release of the tines from the myocardium, allowing withdrawal of the system into the distal cone. RAO, right anterior oblique.

All patients received a new pacing device immediately after the retrieval procedure. When a new TPS was implanted after retrieval, it was generally positioned at the apical septum, slightly above the initial location of the explanted TPS, to avoid reimplantation into any fibrous tissue relate to the old device.

Results

Patient characteristics are shown in Table $\mathbf{1}$

\begin{tabular}{|l|c|l|c|c|l|l|l|}
\hline & $\begin{array}{c}\text { Age, } \\
\text { (year) }\end{array}$ & Gender & $\begin{array}{c}\text { Implant } \\
\text { durations, } \\
\text { (days) }\end{array}$ & $\begin{array}{c}\text { Fluoroscopy } \\
\text { time (min) }\end{array}$ & $\begin{array}{c}\text { Successful } \\
\text { Retrieval }\end{array}$ & \multicolumn{1}{|c|}{$\begin{array}{l}\text { Retrieval } \\
\text { indication }\end{array}$} & $\begin{array}{c}\text { Re-implant } \\
\text { devices }\end{array}$ \\
\hline 1 & 82 & $\mathrm{M}$ & 280 & 37 & Success & Battery depletion & Micra TPS \\
\hline 2 & 54 & $\mathrm{M}$ & 777 & 21 & Success & $\begin{array}{l}\text { Pacemaker } \\
\text { syndrome }\end{array}$ & $\begin{array}{l}\text { Dual chamber } \\
\text { pacemaker }\end{array}$ \\
\hline 3 & 66 & $\mathrm{~F}$ & 406 & 13 & Success & Battery depletion & Micra TPS \\
\hline 4 & 46 & $\mathrm{M}$ & 287 & - & Failure & $\begin{array}{l}\text { Low ejection } \\
\text { fraction }\end{array}$ & CRT-D \\
\hline 5 & 79 & $\mathrm{M}$ & 1219 & 15 & Success & Battery depletion & Micra TPS \\
\hline 6 & 49 & $\mathrm{~F}$ & 360 & 12 & Success & $\begin{array}{l}\text { Pacemaker } \\
\text { syndrome* }\end{array}$ & Micra TPS \\
\hline $\begin{array}{l}\text { Mean, } \\
\text { (SD) }\end{array}$ & $63 \pm 15$ & & $555 \pm 373$ & $19.6 \pm 10.3$ & & & \\
\hline
\end{tabular}

TPS; transcatheter pacing system, CRT; Cardiac Resynchronization Therapy 
The mean duration time from implantation to retrieval was $554 \pm 373$ days (range, 280 to 1219 days). The indication for device retrieval included pacemaker syndrome in two patients, battery depletion due to high pacing threshold and complete dependence of ventricular pacing in three patients, and need for upgrade to biventricular pacing due to the development of low left ventricular ejection fraction from continuous ventricular pacing in one patient.

Successful retrieval, defined as complete removal of the LCP, was achieved in 5 patients $(83 \%)$. The mean procedural fluoroscopy time was $19 \pm 16 \mathrm{~min}$. In all five successful retrieval cases, the proximal retrieval feature could be snared and connected tightly with the delivery catheter. After careful introduction of the distal cone of the delivery catheter over the LCP capsule to cover the full body of the implanted TPS, continuous contraction allowed the tines to be released from the cardiac tissue, and the LCP was pulled back into the distal cone. We carefully inspected all retrieved devices for cardiac tissue remnants, but only blood coagulum was present. No procedure-related adverse device events occurred.

Device retrieval was unsuccessful in one patient. In this patient, the microsnare successfully engaged the proximal retrieval feature of the device; however, even after tight fixation of the snare, we were unable to advance the distal cone over the device. We did not attempt to pull the engaged device because of concern that insufficient countertraction might tear the myocardial tissue. Intraprocedural ICE showed that the Micra TPS was almost embedded within the cardiac wall (Figure 2).

After retrieval, four patients each received a new Micra TPS immediately. All reimplantations were successful and satisfactory electrical parameters were recorded (pacing threshold, $0.57 \pm 0.33 \mathrm{~V}$, sensing threshold at $12.4 \pm 4.5 \mathrm{mV}$; and impedance, $551 \pm 78 \Omega$ ). The remaining two patients received conventional transvenous pacemakers - either dual-chamber or biventricular devices. No procedure-related adverse events occurred during reimplantation. And specifically, no pericardial effusion or tricuspid valve damage was observed on intraoperative ICE.

\section{Discussion}

The primary findings of the present study are as follows: (A) retrieval of chronically-implanted TPS devices was performed safely with currently available endovascular tools, and (B) implantation of a new leadless cardiac pacing device immediately after retrieval of the old one could be a safe and feasible strategy. Although the manufacture recommends abandonment of the TPS at end-of-life, its retrieval may be preferred in specific scenarios. However, there are no detailed studies regarding the safety and feasibility for retrieval and replacement of chronicallyimplanted TPS devices. In the present study, retrieval was performed only after a clear discussion with patients regarding the basic principle of the options of retrieval or abandonment of the TPS. If the retrieval attempt was unsuccessful, it was planned to abandon the old TPS and implant the appropriate new cardiac pacing device.

First, although there is no dedicated system for the retrieval of a chronically implanted TPS devices, retrieval could be performed safely with currently available tools. However, there are several clinical implications including the possibility for elective reimplantation of a new LCP, reductions in the risk of potential device-to-device interactions and long-term risks of multiple devices implanted in the RV. Although acute retrievability has been reported, to the best of our knowledge, this is the first study to examine the safety and feasibility of retrieval of chronicallyimplanted TPS devices. According to a careful review of the literature (see Table 2), there are several case reports of TPS retrieval - totalling 29 retrieval patients with explantation performed within 100 days of the initial implantation procedure. Among the five retrieval cases which were performed after 100 days from initial implantation, three were retrieved successfully $(60 \%)$. In contrast, in our study, all patients had the TPS implanted for at least 280 days and the longest implantation period was 1219 days.

\begin{tabular}{|c|c|c|c|c|c|c|c|}
\hline Study & Publish & Number & Age, $y$ & $\begin{array}{l}\text { Implant } \\
\text { durations }\end{array}$ & $\begin{array}{l}\text { Retrieval } \\
\text { Success }\end{array}$ & Retrieval indication & $\begin{array}{l}\text { Re-implant } \\
\text { device }\end{array}$ \\
\hline Fichtner et al [7] & 2019 & 1 & 83 & 1 day & $100 \%$ & Dislodgement & VVI \\
\hline Curnis et al [11] & 2019 & 1 & 41 & 29 months & $100 \%$ & Battery depletion & TPS \\
\hline Morita et al [12] & 2018 & 1 & 74 & 0 day & $100 \%$ & Dislodgement & TPS \\
\hline Morani et al [13] & 2017 & 1 & 80 & 40 days & $100 \%$ & Dislodgement & N/A \\
\hline Karim et al [14] & 2015 & 1 & 61 & 21 days & $100 \%$ & High pacing threshold & TPS \\
\hline Koay et al [15] & 2016 & 1 & 80 & 1 month & $100 \%$ & Infection & N/A \\
\hline Kiani et al [16] & 2019 & 1 & 78 & 4 years & $100 \%$ & Pacemaker syndrome & CRT-D \\
\hline Nozoe et al [17] & 2018 & 1 & 86 & 56 & $100 \%$ & Infection suspicious & N/A \\
\hline $\begin{array}{l}\text { Grubman et al } \\
{[18]}\end{array}$ & 2017 & 5 & $43-67$ & 5 - 406 days & $60 \%$ & N/A & TPS \\
\hline \multirow[t]{2}{*}{ Afzal et al [10] } & 2018 & 11 & N/A & 0 day & $100 \%$ & N/A & N/A \\
\hline & & 18 & N/A & 1 - 95 days & $100 \%$ & N/A & N/A \\
\hline
\end{tabular}

TPS; transcatheter pacing system

Table 2: Case examples of the retrieval of Micra transcatheter pacing system

In our experience reimplantation of a new TPS immediately after retrieval was safe and effective in patients who require replacement due to end of battery life or other reasons. In the four patients who received a new TPS immediately after retrieval, all reimplantation procedures were performed safely without any procedure-related complications and with excellent electrical measurements. Reimplantation of a new TPS after retrieval may be preferable to traditional transvenous device systems because an LCP can prevent transvenous lead- and pocket-related complications, and requires only one right femoral vein puncture for both retrieval and reimplantation.

Retrieval was unsuccessful in only one of six patients. In this patient, although the proximal retrieval feature of the TPS could be engaged by 
the snare, detaching the TPS from the myocardium was not possible. Notably, in this case, the ICE imaging revealed that the TPS was embedded into the ventricular myocardium (Figure 2). Reports describing adherent tissue surrounding an implanted TPS device are limited. One autopsy study of the patient with an implanted TPS showed device encapsulation [8]. Another report noted a TPS device totally covered with fibrous tissue at autopsy less than one year after implantation [9]. The severity of embeddedness within fibrous tissue might be associated with the difficulty of TPS retrieval. ICE imaging appears helpful not only for the retrieval manipulation of the snare and the early detection of pericardial effusion during retrieval but also for the assessment of the retrieval difficulty.

Two approaches can be utilized for retrieval of the TPS (Figure 3-A, 3B).

\section{B}

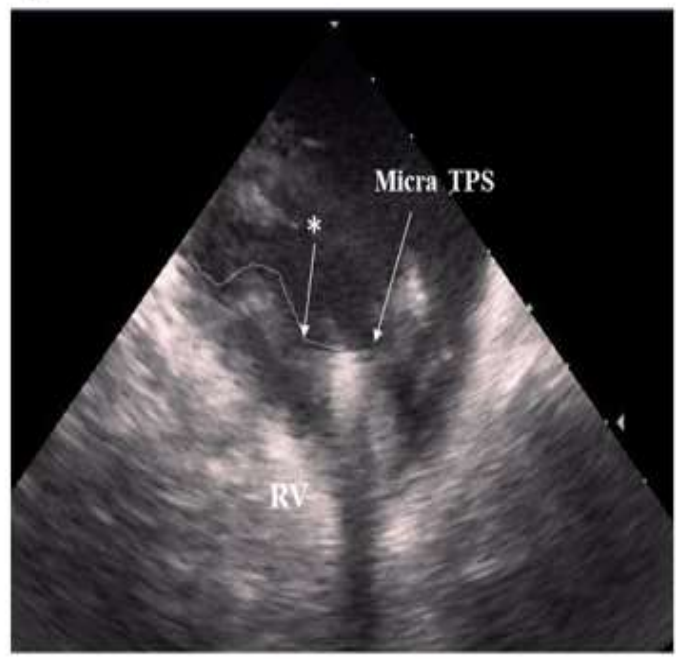

Figure 3: Intracardiac echocardiography (ICE). A) ICE in a case of successful retrieval of the Micra transcatheter pacing system (TPS). The proximal retrieval feature is visible, and the Micra TPS was not covered by surrounding tissue. B) ICE in a case of unsuccessful retrieval. Surrounding tissue was severely adherent to the Micra TPS, which was almost embedded within the cardiac wall (*denoted by the asterisk). RV, right ventricle.

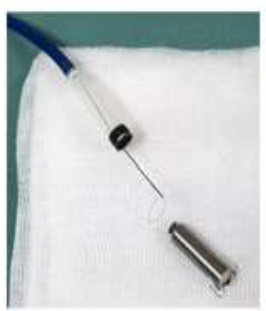

A-1

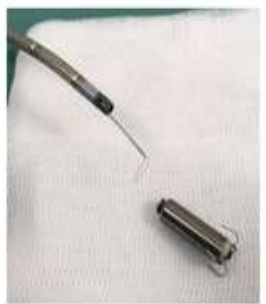

B-1

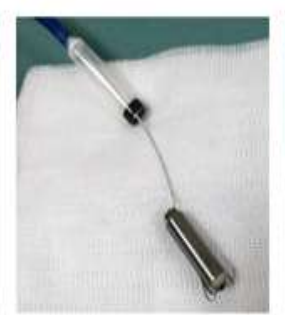

A-2

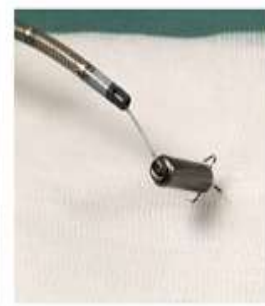

B-2

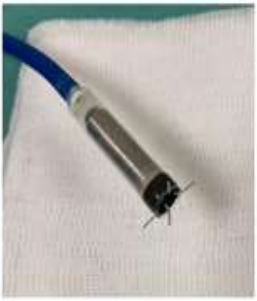

A-3

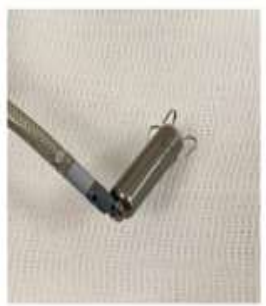

B-3

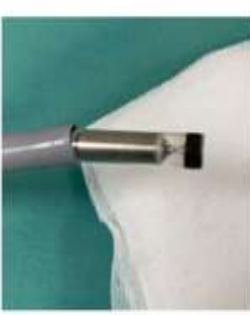

A-4

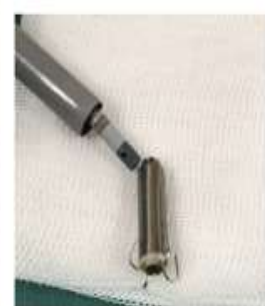

B-4

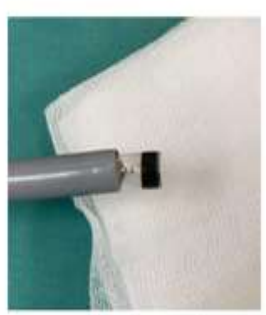

A-5

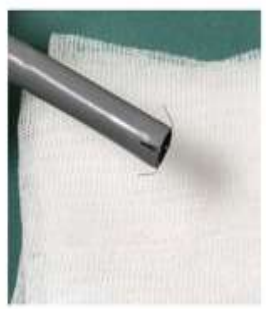

B-5

Figure 4. Two approaches for retrieval of Micra transcatheter pacing system (TPS) retrieval: A) Micra delivery catheter plus snare method and B) steerable sheath plus snare method. A-1) A single-loop $7 \mathrm{~mm}$ snare wire is inserted through the central lumen of the Micra delivery catheter introduced through Micra sheath. The system's distal cone is positioned at the proximal aspect of the device. After snare advancement, the catheter is deployed around the proximal retrieval feature of the Micra transcatheter pacing system (TPS). A-2) The snare is engaged and locked around the retrieval feature the Micra TPS. A-3) Tension on the snare along with countertraction from the distal cone results in release of the tines from the myocardium and the system is withdrawn fully back into the distal cone. A-4 \& A-5) The delivery catheter and Micra TPS are withdrawn into the introducer sheath and removed from the body. B-1) A snare is inserted through a steerable sheath, which can accommodate snare size up to 20 mm. B-2) Engaging the snare to the proximal retrieval feature. B-3) Traction in the snare causes Micra TPS detachment from the myocardium. B-4 \& $B-5)$ Pulling back the sheath and Micra TPS into the outer sheath. 
Both approaches require femoral venous access with insertion of a TPS introducer sheath, but then diverge with regard to the specific tool employed to engage the TPS during the next step: the snare is advanced through either i) the integrated protectable sleeve of the TPS delivery catheter (as employed in our case series), or ii) a steerable sheath [10]. In using a steerable sheath, a short sheath (11-16 Fr) is first inserted into the introducer sheath to prevent back bleeding, and a steerable sheath (for example, 8.5 Fr, Agilis NXT, Abbott Laboratories, Abbott Park, IL, USA) is inserted and advanced into the RV.

The differences between the two approaches are the acceptable snare size, and the ability to apply countertraction force to detach the TPS from the myocardium. That is, it is easier to snare the retrieval feature using a steerable sheath, because this sheath allows the use of a $20 \mathrm{~mm}$ loop diameter snare (or a tri-loop snare if desired). However, the steerable sheath is limited by the inability to withdraw the LP into the steerable sheath due to its smaller diameter. In contrast, the TPS delivery catheter only accommodates a $7 \mathrm{~mm}$ snare; this snare size is small, but still feasible to engage the proximal retrieval feature of the TPS. On the other hand, this approach allows the ability to provide true countertraction using the distal cone. Indeed, because of the chronic nature of the TPS implantations, leading to our expectation of encountering fibrous tissue intimately associated with the device, we used the TPS delivery system with the $7 \mathrm{~mm}$ loop snare for all of our retrieval cases. Finally, one practical limitation of this latter approach is the need for a TPS delivery catheter. This can be accomplished by first placing a new TPS device in the heart, and then using the same deliver catheter to retrieve the old LCP. Of course, it would be the most ideal if a dedicated retrieval kit was developed address some of the practical limitations of the current off-theshelf tools approaches, as well as the fibrous tissue adhesions surrounding the device that can prevent successful retrieval.
Based on our experiences, retrieval of chronically implanted TPS devices can be performed safely, and immediate reimplantation of a new leadless cardiac pacing device is feasible. Using ICE during retrieval might play a critical role not only in the rapid detection of pericardial effusion but also in the assessment of the retrieval difficulty. Our findings may overcome the concern of potential multiple intracardiac devices in patients with long life expectancy and allow more extensive use of LPs. A worldwide registry and future studies regarding retrieval of chronically-implanted Micra TPS devices should be implemented to better elucidate its safety and efficacy.

Limitations: This study has several limitations. First, it is a nonrandomized observational single-center experience with a small sample size. Second, the learning curve for the procedure may have influenced the fluoroscopy time and retrieval success rate. However, all retrieval procedures in this study were performed by two experienced operators. Third, it is important to recognize that the meantime from implantationto-explantation was only $\sim 2$ tears, with the longest duration being just over three years. One cannot conclude from the experience that retrieval success will remain in the $80 \%$ range when even longer follow-up times. Finally, the difficulty of retrieving an infected TPS devices remains unclear, although based on clinical experience with lead extraction, if anything, infection should facilitate retrieval.

\section{Conclusions}

The retrieval of a long-standing implanted Micra TPS device was safely performed, which may indicate the possibility of their safe and elective replacement with a new leadless cardiac pacing device.

\section{Supplemental Materials}

Online Video A, B, C






\section{. \\ Video A.mp4}

Online Video A. Approaching the retrieval system to the proximal aspect of the Micra transcatheter pacing system. The retrieval system (A singleloop $7 \mathrm{~mm}$ snare wire and the delivery sheath of Micra transcatheter pacing system (TPS)) was advanced under fluoroscopy and intracardiac echocardiography to the right atrium. The system's distal cone was manoeuvred into the right ventricle (RV) to the proximal aspect of the TPS device.
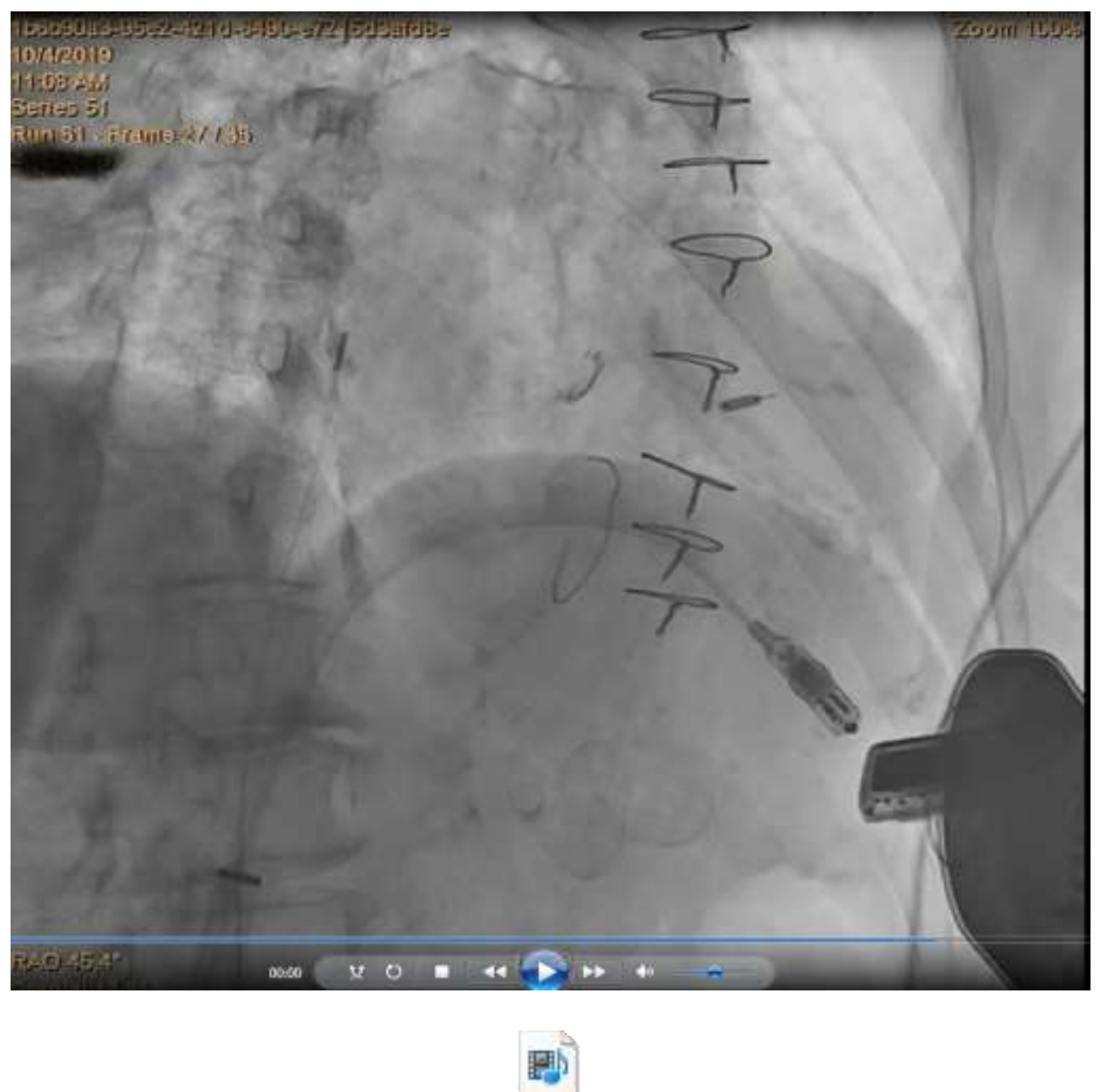

\section{Video B.mp4}

Online Video B. Docking the retrieval system and Micra transcatheter pacing system. A single snare was deployed around the proximal retrieval feature of the Micra transcatheter pacing system (TPS). After confirming coaxial alignment between the snare and the retrieval feature with multiplane fluoroscopy, the snare was closed and locked around the retrieval feature of TPS. 



\section{Video C.mp4}

Online Video C. Releasing a Micra transcatheter pacing system from the myocardium. A snare loop was tightened to hold the device firmly, and tension was applied, along with countertraction force from the distal cone, to release the tines from the myocardium, allowing withdrawal of the device into the distal cone. The delivery catheter and Micra TPS were then withdrawn into the introducer sheath.

\section{Acknowledgement}

Drs Neužil and Reddy were the leading operators for retrieval of the TPS device. Drs Neužil and Minami took part in data evaluation and writing this manuscript; Dr. Reddy participated in critical revisions of the manuscript. All co-authors contributed fully terms of the design of the study, the evaluation of data, the actual manuscript preparation. The corresponding author confirms that all authors have seen and approved the final text.

\section{References}

1. Reynolds D, Duray GZ, Omar R, et al. A leadless intracardiac transcatheter pacing system. N Engl J Med. 2016; 374:533-541.

2. Reddy VY, Exner DV, Cantillon DJ, et al. Percutaneous implan tation of an entirely intracardiac leadless pacemaker. N Engl J M ed. 2015;373:1125-1135.

3. Roberts PR, Clementy N, Al Samadi F, et al. A leadless pacemaker in the real-world setting: The Micra Transcatheter Pacing System Post-Approval Registry. Heart Rhythm. 2017; 14:1375-1379. 
4. Reddy VY, Miller MA, Knops RE, et al. Retrieval of the leadles s cardiac pacemaker: A multicenter experience. Circ Arrhythm Electrophysiol 2016; 9:e004626.

5. Lakkireddy D, Knops R, Atwater B, et al. A worldwide experie nce of the management of battery failures and chronic device ret rieval of the Nanostim leadless pacemaker. Heart Rhythm. 2017 ;14:1756-1763.

6. Minami K, Neuzil P, Petru J, et al. Retrieval of Long-Term Imp lanted Leadless Pacemakers: A Single-Center Experience. J Am Coll Cardiol EP. 2020; doi.org/10.1016/j.jacep.2020.06.010.

7. Fichtner S, Estner HL, Näbauer M, Hausleiter J. Percutaneous e xtraction of a leadless Micra after dislocation: A case report Eur Heart J Case Rep. 2019;13;3(3): ytz113.

8. Vamos M, Honold J, Duray GZ, Hohnloser SH. MICRA Leadless Pacemaker on Autopsy. JACC Clin Electrophysiol. 2016; 2:636637.

9. Kypta A, Blessberger H, Lichtenauer M, Steinwender C. Complete encapsulation of a leadless cardiac pacemaker. Clin Res Cardiol. 2015; 105:94.

10. Afzal MR, Daoud EG, Cunnane R, et al. Techniques for successful early retrieval of the Micra transcatheter pacing system: a worldwide experience. Heart Rhythm. 2018; 15:841846.
11. Curnis A, Cerini M, Mariggiò D, et al. First-in-human retrieval of chronically implanted Micra transcatheter pacing system. Pacing Clin Electrophysiol. 2019;42:1063-1065

12. Morita J, Fukunaga M, Hiroshima K, et al. Retrieval of a Micra transcatheter pacing system in a heart with a preexisting lead. Indian Pacing Electrophysiol. 2018;18:183-184.

13. Morani G, Bolzan B, Tomasi L, et al. Troubleshooting in PM Leadless: How to manage an indissoluble knot. J Arrhythm. 2019;35:676-678.

14. Karim S, Abdelmessih M, Marieb M, Reiner E, Grubman E. Extraction of a Micra transcatheter pacing system: first-inhuman experience. HeartRhythm Case Rep. 2016;2:60-62.

15. Koay A, Khelae S, Wei KK, Muhammad Z, Ali RM, Omar R. Treating an infected transcatheter pacemaker system via percutaneous extraction. HeartRhythm Case Rep. 2016;2:360362.

16. Kiani S, Merchant FM, El-Chami MF. Extraction of a 4-year-old leadless pacemaker with a tine-based fixation. HeartRhythm Case Rep. 2019;5:424-425.

17. Nozoe M, Yoshida D, Nagatomo D, et al. Successful percutaneous retrieval of a micra transcatheter pacing system at 8 weeks after implantation. J Arrhythm. 2018;34:653-655.

18. Grubman E, Ritter P, Ellis CR, et al. To retrieve, or not to retrieve: System revisions with the Micra transcatheter pacemaker. Heart Rhythm. 2017;14:1801-1806.
This work is licensed under Creative Commons Attribution 4.0 License

To Submit Your Article Click Here: Submit Manuscript

DOI:10.31579/2641-0419/0138
Ready to submit your research? Choose Auctores and benefit from:

* fast, convenient online submission

* rigorous peer review by experienced research in your field

* rapid publication on acceptance

* authors retain copyrights

* unique DOI for all articles

* immediate, unrestricted online access

At Auctores, research is always in progress.

Learn more www.auctoresonline.org/journals/clinical-cardiology-andcardiovascular-interventions 